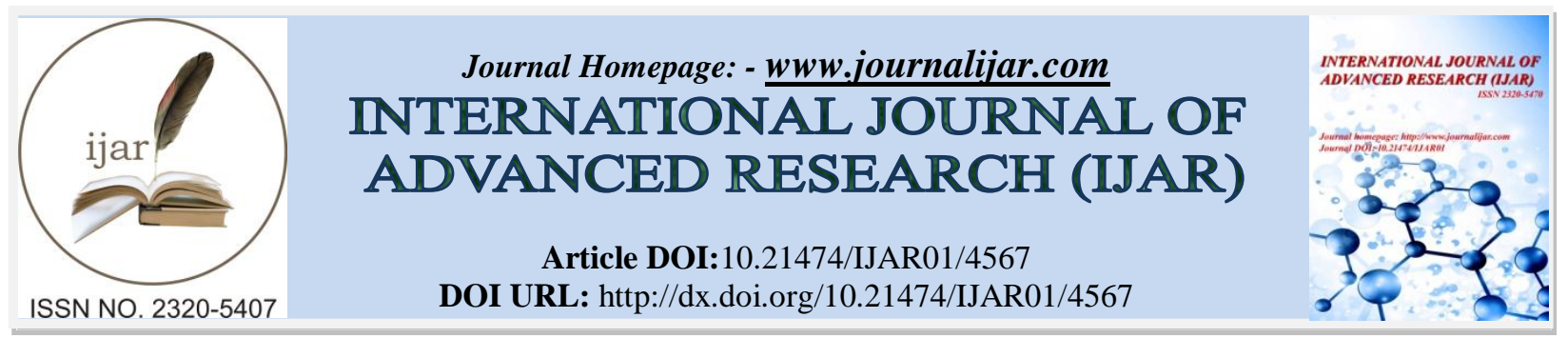

RESEARCH ARTICLE

\title{
MOLECULAR INTERACTION OF NON-STEROID ANTI-INFLAMMATORY DRUG ACECLOPHENAC WITH LEUCINE IN DMSO MEDIUM: AN ULTRASONIC STUDY.
}

\author{
Sunil Kumar Pradhan ${ }^{1}$, $S m r u t i$ Prava Das ${ }^{2}$, Ashok Kumar Sahoo ${ }^{3}$, Monalisa Das ${ }^{4}$ and Ajaya Kumar \\ Patnaik2. \\ 1. Department of Chemistry, Brahmani College,Dandisahi,Kendrapara, Odisha -754240, India. \\ 2. Department of Chemistry, Ravenshaw University, Cuttack, Odisha-753003, India. \\ 3. Department of Chemistry, Christ College, Cuttack, Odisha-753008, India. \\ 4. Department of Chemistry, Govt. Women's College, Keonjar-758001.
}

\section{Manuscript Info}

Manuscript History

Received: 22 April 2017

Final Accepted: 24 May 2017

Published: June 2017

Key words:-

adiabatic compressibility, intermolecular free length, acoustic impedance, apparent molar volume, apparent molar compressibility, ion-dipole interaction.

\section{Abstract}

Non steroidal anti-inflammatory drug aceclophenac has hydrophobic and hydrophilic domain. It is sparingly soluble in water and miscible in blood due to hydrophilic nature. It exhibits analgesic, antipyretic and anti-inflammatory activities. It is best used in rheumatoid arthritis. It is inhibitor of clotting in the blood vessel, thus prevents heart attack and stroke. Leucine is an essential $\alpha$ - amino acid used in biosynthesis of protein and also used for muscle building. It contains $\alpha$ - amino group, carboxylic acid group and an isobutyl side chain. Ultrasonic study of Aceclophenac with Leucine in DMSO medium provides much useful information about the nature of their molecular interaction in a polar aqua-organic medium. In our body drug interaction with protein play very vital role in the biological process. Because of the 3D structure of protein, it is quite difficult to study its interaction with drugs in the biological system. As amino acid is a model component for protein, in this piece of work we have studied the drug amino acid molecular interaction with variation of concentration of amino acid ranging from $0.002-0.01 \mathrm{~mol} / \mathrm{kg}$ and the drug concentration varies from $0.0002-0.001 \mathrm{~mol} / \mathrm{kg}$. The different acoustic parameters like adiabatic compressibility $(\beta)$, intermolecular free length $\left(\mathrm{L}_{\mathrm{f}}\right)$, acoustic impedance $(\mathrm{z})$, apparent molar volume $\left(\mathrm{V}_{\varphi}\right)$,apparent molar compressibility $\left(\mathrm{K}_{\varphi}\right)$,partial molar volume $\left(\mathrm{V}_{\varphi}{ }^{\mathrm{o}}\right)$,partial molar compressibility $\left(\mathrm{K}_{\varphi}{ }^{\circ}\right)$,John Dole coefficient A,B are calculated from density $(\rho)$, ultrasonic velocities (U) and viscosity $(\eta)$ data. The parameters are calculated at $298.15 \mathrm{~K}$ in a polar aprotic medium i.e. taking DMSO as solvent are interpreted in terms of ion-solvent, ionion and ion-dipole interactions considering the structure breaking and structure making aspects of the solvent. 


\section{Introduction:-}

Ultrasonic studies of drugs find extensive application in solution chemistry as speed of sound in liquid and liquid mixture intrinsically related to parameters which characterize physico-chemical behaviour. Physico-chemical properties such as density, viscosity, and speed of sound have considerable importance in building theoretical model.Ultrasonic studies has also find importance in petro-chemical, pharmaceutical and designing instruments for study in these fields [1]. Research and experimental data obtained from this are used as excellent tools to detect solute-solute and solvent interaction. Recently few papers have been reported by some researchers regarding ultrasonic and thermodynamic study of various drugs in different solvent medium and mix solvent medium.[ 26]But very little work has been done on the interaction of drug-amino acids. Amino acids are important biomolecules through which the drugs interact inside the body. The interaction of drug with amino acid in different solvent medium can be better studied through ultrasonic technique. This prompted us to do this piece of work on drug-amino acid interaction in DMSO medium.[7,8] Solvent-mediated interactions in biological systems constitute the main determinants for regulating the structure, dynamics, and thermodynamics of bio-molecules, ultimately dictating their biological function and that of the cell. Because bio-molecules are embedded in a highly polar medium, electrostatics is a fundamental component of solvation effects at the molecular level, both in the context of the cell and in the test tube. This is a piece of comprehensive study on molecular interaction of drug with amino acids in a polar aqua-organic medium by evaluating volumetric, viscometric and spectroscopic properties.

* Aceclophenac is a non steroid anti inflammatory drug. It is commonly called as Phenyl Acetoxy Acetic Acid. The IUPAC name of Aceclophenac is (2-[2, 6 dichlorophenyl) amino] phenyl acetic acid. Aceclophenac is used for treatment of rheumatoid arthritis, Osteo arthritis, Ankylosing spondylitis, tendinitis, and joint inflammation and reduces pain intensity and duration of morning stiffness and other inflammatory conditions . [9,10]

* Leucine is an essential amino acid required by our body.It is not synthesized in our body. Leucine interacts with insulin signaling pathway to stimulate control of protein synthesis. Maintenance of muscle protein during period of restricted energy intake. Reduce postprandial glucose and insulin maintaining blood glucose level. $[11,12]$

* The physicochemical properties of bio molecule and drugs in different solvent medium are very much significant for knowing their binding trends. This also helps in understanding mechanism of drugs and amino acids in body system. The interaction of amphiphilic molecules [drugs] with amino acids through thermo physical parameters has been studied.[13-15]

* DMSO is a polar aprotic non reacting solvent. Both Leucine and Aceclophenac are soluble in DMSO. It is membrane penetrating water repellant. It is used for in vitro and in vivo studies. It is used peripheral nerve degeneration and drug delivery.[16,17]

* The pharmacokinetics and pharmacodynamic properties such as diffusion, transmission and absorption of the drugs are observed as path determining results of drugs and functionally active bio-molecules like amino acids. $[18,19,20,21]$

\section{Experimental:-}

A sample of Aceclophenac was procured from Sigma Aldrich Company of $99.9 \%$ purity.Leucine and DMSO of AR grade were also procured from Sigma Aldrich Company with $99.9 \%$ purity. Ultrasonic Velocity was measured by Ultrasonic interferometer (M-81-S) model at $2 \mathrm{MHz}$ frequency.All measurements were done under atmospheric condition. The density measurement was done by densitometer (Anton par DSA 5000 model) with a resolution of 10 ${ }^{6} \mathrm{gm} \mathrm{cm}^{-3}$ and viscosity was measured in Oswald's viscometer distilled by calibrating with doubly distilled water with an accuracy of $+0.001 \mathrm{~Pa} \mathrm{Sec}$. All the measurements were performed at $303.15 \mathrm{~K}$. Before use all the chemicals were dried for $48 \mathrm{hr}$ under reduced pressure at $303.15 \mathrm{~K}$ and then stored in a desiccator over silica gel. The chemicals were weighed in an electronic digital balance (SHIMADZU AX- 200) Japan with a least count of 0.0001 gm. The Aceclophenac and Leucine in a ratio 1:1 in DMSO was refluxed for 72 hour. The IR of product was taken. The IR study was carried out in Nicolas IS-5 model IR. The UV-VIS study was carried out in Agilent carry 100 model double beam spectrophotometer. Ultrasonic and volumetric parameters were determined applying following equations.

Adiabatic compressibility $(\beta)$,

$\beta=d^{-1} U^{-2}$

Apparent molar volume $\left(\mathrm{V}_{\varnothing}\right)$,

$V_{\phi}=1000\left(c d_{0}\right)^{-1}\left(d_{0}-d\right)+M_{2} d_{0}^{-1}$ 
$V_{\phi}=V_{\phi}^{0}+S_{v} c^{1 / 2}$

Apparent molar compressibility $\left(K_{\phi}\right)$

$K_{\phi}=1000 \beta c^{-1}-\beta^{0} d^{-1}\left(1000 c^{-1}-M_{2}\right)$

$K_{\phi}=K_{\phi}{ }^{0}+S_{k} c^{1 / 2}$

The viscosity parameters are calculated using John Dole equation

Acoustic Impedance $(Z)$

$$
\eta_{r}=\frac{\eta}{\eta_{0}}=1+A c^{1 / 2}+B c
$$

Intermolecular Free length $\left(L_{f}\right)$

$$
Z=U d
$$

$L_{f}=K_{j} \beta^{1 / 2}$

Where $K_{j}$ is Jacobson constant $=2.0965 \times 10^{-6}$

The John Dole Parameter $A$ and $B$ can be calculated using the relationship

$$
\eta_{r}=\frac{\eta}{\eta_{0}}=1+A c^{1 / 2}+B c
$$

$A$ is Falkenhagen coefficient represent solute-solute interaction. In this experiment the positive value A indicates Aceclophenac -Leucine interaction in DMSO. The decrease of negative value of $B$ co- efficient value is found to be negligible indicating weak drug solvent interaction.

Table 1:- Infrared data of LEU, ACE and ACE-LEU complex.

\begin{tabular}{|l|l|l|l|l|l|}
\hline compound & \multicolumn{5}{|c|}{ IR frequencies $\left(\mathrm{cm}^{-1}\right)$} \\
& $v_{O H}$ & $v_{C=O}$ & $v_{C-O-C}$ & $v_{N H_{2}}$ & $v_{C-N}$ \\
\hline LEU & - & - & 1406 and 1581 & 2957 & - \\
\hline ACE & 3331 & 1771 & $1050-1178$ & - & - \\
& & & $1178-1280$ & & 1435 \\
\hline ACE-LEU & - & 1730 & & - & \\
\hline
\end{tabular}

Table 2:- Physical data of Aceclophenac at different concentration of Leucine..

\begin{tabular}{|l|l|l|c|}
\hline $\begin{array}{l}\text { Concentration of } \\
\text { Aceclophenac }\end{array}$ & Ultrasonic Velocity(U) & Density(d) & Viscosity $\left(\eta_{r}\right)$ \\
\hline & \multicolumn{1}{|c|}{ Leucine=0.0002 } & 1.00070 & 0.9223 \\
0.002 & 1488.2 & 1.00075 & 0.9254 \\
\hline 0.004 & 1486.9 & 1.00081 & 0.9275 \\
\hline 0.006 & 1485.7 & 1.00086 & 0.9301 \\
\hline 0.008 & 1483.8 & 1.00092 & 0.9327 \\
\hline 0.01 & 1482.7 & 1.00079 & 0.9273 \\
\hline & \multicolumn{1}{|c|}{ Leucine=0.0004 } & 0.9298 \\
\hline 0.002 & 1490.3 & 1.00085 & 0.9323 \\
\hline 0.004 & 1486.5 & 1.00091 & 0.9350 \\
\hline 0.008 & 1482.4 & 1.00102 & 0.9381 \\
\hline 0.01 & 1478.3 & 1.00109 & 0.9285 \\
\hline \multicolumn{2}{|c|}{1470.2} & 1.00081 & 0.9313 \\
\hline
\end{tabular}




\begin{tabular}{|c|c|c|c|}
\hline 0.004 & 1480.1 & 1.00223 & 0.9340 \\
\hline 0.008 & 1475.2 & 1.00295 & 0.9366 \\
\hline 0.01 & 1769.3 & 1.00368 & 0.9388 \\
\hline \multicolumn{4}{|c|}{ Leucine=0.0008 } \\
\hline 0.002 & 1498.6 & 1.00087 & 0.9347 \\
\hline 0.004 & 1488.3 & 1.00096 & 0.9380 \\
\hline 0.006 & 1484.4 & 1.00135 & 0.9399 \\
\hline 0.008 & 1480.2 & 1.00184 & 0.9427 \\
\hline 0.01 & 1472.6 & 1.00267 & 0.9455 \\
\hline \multicolumn{4}{|c|}{ Leucine=0.001 } \\
\hline 0.002 & 1500.2 & 1.00089 & 0.9421 \\
\hline 0.004 & 1488.5 & 1.00124 & 0.9446 \\
\hline 0.006 & 1482.6 & 1.00231 & 0.9527 \\
\hline 0.008 & 1475.3 & 1.00297 & 0.9560 \\
\hline 0.01 & 1416.4 & 1.00352 & \\
\hline
\end{tabular}

Table 3:- Measured parameters of Aceclophenac at different concentration of leucine.

\begin{tabular}{|c|c|c|c|c|c|}
\hline $\mathrm{w}$ & $\beta \times 10^{-7} \mathrm{~m}^{2} \mathrm{~N}^{-1}$ & $\begin{array}{c}\mathrm{L}_{\mathrm{f}} \times 10^{-7} \\
\mathrm{~m}\end{array}$ & $\begin{array}{c}Z \times 10^{9} \\
\mathrm{Kg} \mathrm{m}^{2} \mathrm{~s}^{-1}\end{array}$ & $\begin{array}{c}K_{\phi} \\
\mathrm{m}^{2} \mathrm{~N}^{-1}\end{array}$ & $\begin{array}{c}V_{\phi} \\
\mathrm{m}^{3} \mathrm{~mol}^{-1}\end{array}$ \\
\hline \multicolumn{6}{|c|}{ Leucine-0.0002 } \\
\hline 0.002 & 4.51204 & 43.97 & 1489.2417 & 4.19191 & 41.477 \\
\hline 0.004 & 4.51971 & 44.01 & 1488.0151 & 4.19904 & 20.903 \\
\hline 0.006 & 4.52674 & 44.04 & 1486.9031 & 4.20557 & 14.043 \\
\hline 0.008 & 4.53811 & 44.09 & 1485.0761 & 4.21614 & 10.614 \\
\hline 0.01 & 4.54458 & 44.12 & 1484.0641 & 4.22229 & 8.556 \\
\hline \multicolumn{6}{|c|}{ Leucine-0.0004 } \\
\hline 0.002 & 4.49893 & 43.91 & 1491.4773 & 4.17973 & 41.432 \\
\hline 0.004 & 4.52169 & 44.01 & 1487.7635 & 4.20088 & 20.878 \\
\hline 0.006 & 4.54646 & 44.13 & 1483.7489 & 4.22391 & 14.021 \\
\hline 0.008 & 4.57121 & 44.25 & 1471.6996 & 4.24691 & 10.594 \\
\hline 0.01 & 4.62141 & 44.49 & 1471.8025 & 4.29353 & 8.539 \\
\hline \multicolumn{6}{|c|}{ Leucine-0.0006 } \\
\hline 0.002 & 4.47658 & 43.79 & 1495.2101 & 4.15897 & 41.422 \\
\hline 0.004 & 4.52662 & 44.04 & 1487.4426 & 4.20551 & 20.713 \\
\hline 0.006 & 4.55414 & 44.17 & 1483.4006 & 4.23111 & 13.806 \\
\hline 0.008 & 4.58161 & 44.31 & 1479.5518 & 4.25666 & 10.353 \\
\hline 0.01 & 4.61512 & 44.46 & 1474.7071 & 4.28598 & 8.281 \\
\hline \multicolumn{6}{|c|}{ Leucine-0.0008 } \\
\hline 0.002 & 4.44888 & 43.66 & 1499.9037 & 4.13324 & 41.392 \\
\hline 0.004 & 4.51026 & 43.96 & 1489.7376 & 4.19027 & 20.850 \\
\hline 0.006 & 4.53223 & 44.06 & 1486.5041 & 4.21071 & 13.953 \\
\hline 0.008 & 4.55575 & 44.18 & 1482.9235 & 4.23258 & 10.492 \\
\hline 0.01 & 4.59909 & 44.39 & 1476.5318 & 4.27365 & 8.381 \\
\hline \multicolumn{6}{|c|}{ Leucine-0.001 } \\
\hline 0.002 & 4.43930 & 43.610 & 1501.5351 & 4.12434 & 41.382 \\
\hline 0.004 & 4.50779 & 43.940 & 1490.3457 & 4.18799 & 20.780 \\
\hline 0.006 & 4.53889 & 44.100 & 1486.0248 & 4.21694 & 13.793 \\
\hline 0.008 & 4.58090 & 44.304 & 1479.6816 & 4.25601 & 10.351 \\
\hline 0.01 & 4.56709 & 46.135 & 1421.3857 & 4.61484 & 8.296 \\
\hline
\end{tabular}


Table 4:- calculated values of Falkenhagen coefficient $(A)$ and Jone- Dole coefficient $(B)$

\begin{tabular}{|c|c|c|}
\hline $\begin{array}{c}\text { Leucine conc. } \\
\text { moledm }\end{array}$ & $\begin{array}{c}A \\
\mathrm{dm}^{3 / 2} \mathrm{~mol}^{-1 / 2}\end{array}$ & $\begin{array}{c}B \\
\mathrm{dm}^{3} \mathrm{~mol}^{-1}\end{array}$ \\
\hline 0.0002 & 1.905 & -12.80 \\
\hline 0.0004 & 1.983 & -13.36 \\
\hline 0.0006 & 2.006 & -13.52 \\
\hline 0.0008 & 2.113 & -14.28 \\
\hline 0.001 & 2.218 & -14.77 \\
\hline
\end{tabular}

Figures:

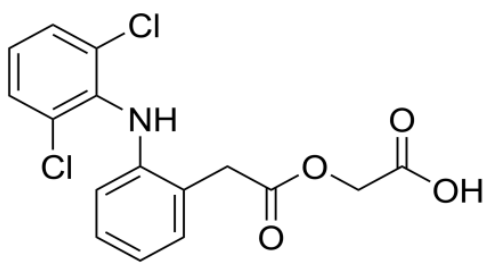

Figure 1:- structure of aceclophenac.

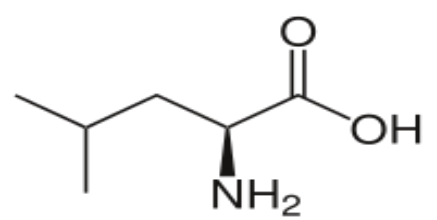

Figure 2:- structure of Leucine.

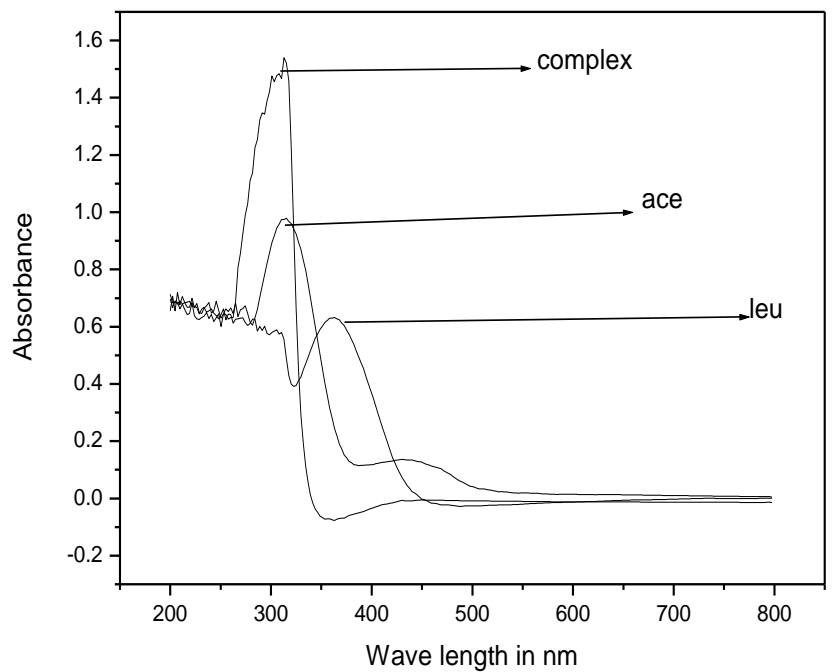


Figure 3:- Comparative UV-VIS Spectra of ACE, LEU and ACE-LEU Complex in DMSO Medium.

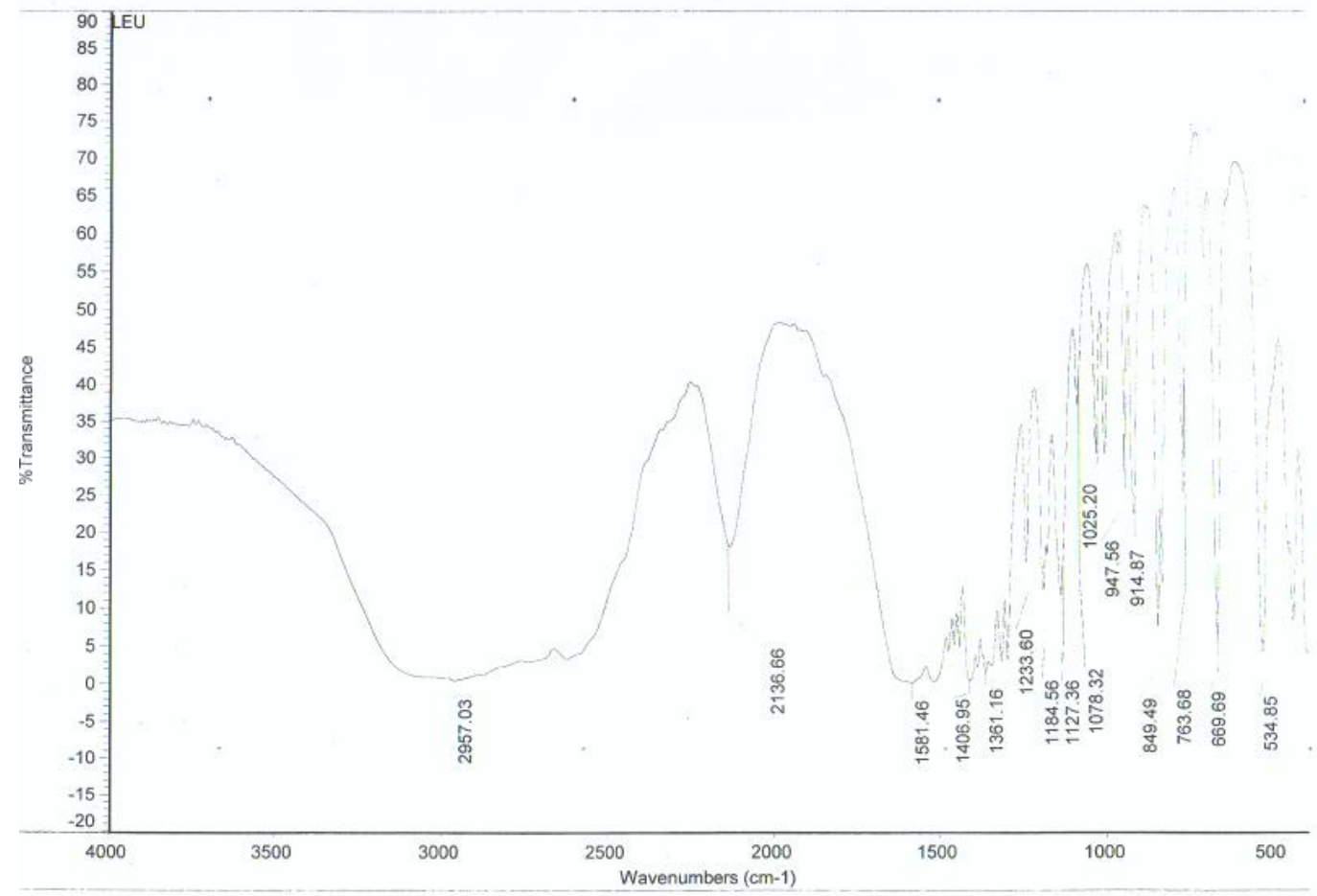

Figure 4:- FTIR spectra of leucine.

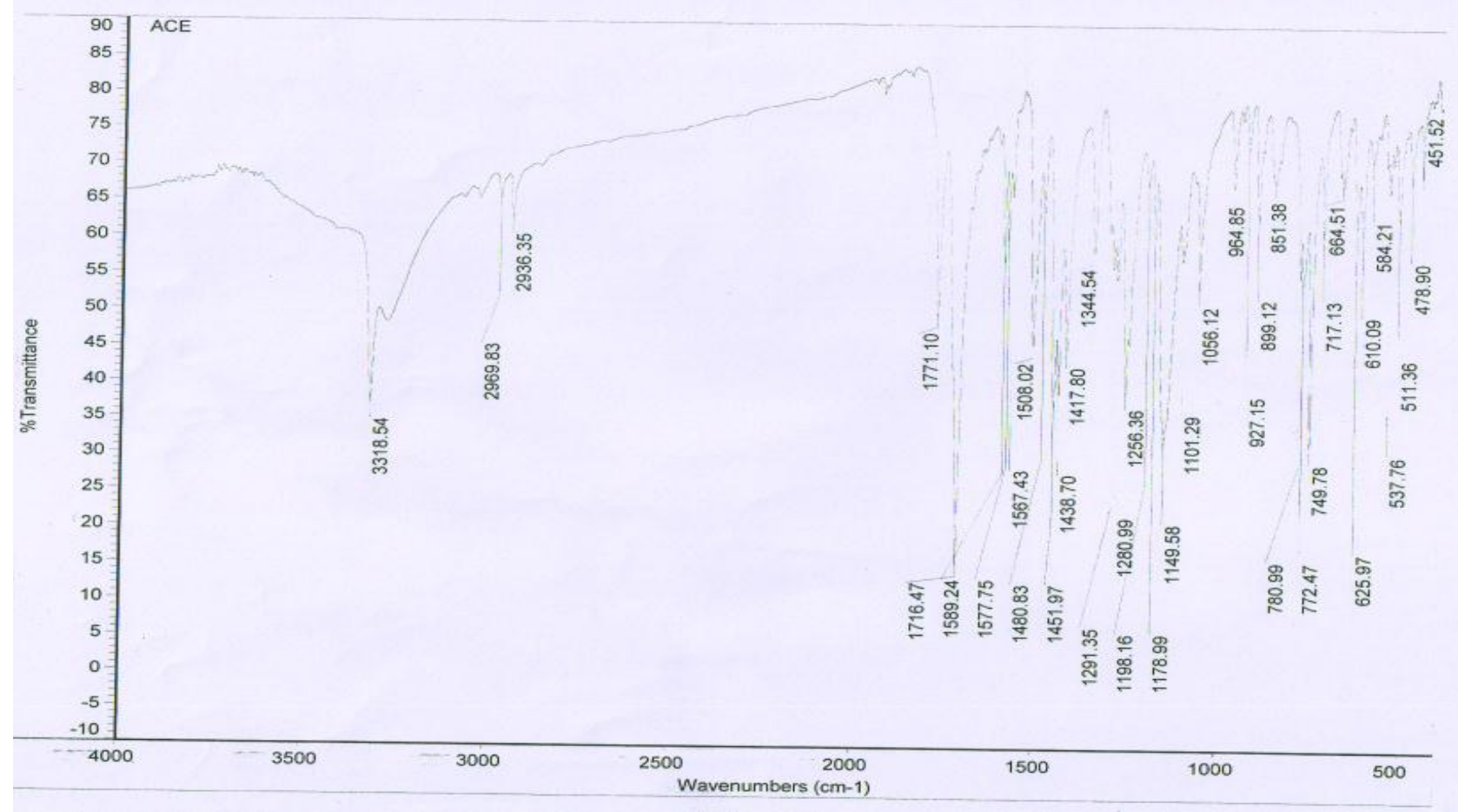

Figure 5:- FTIR spectra of Aceclophenac. 


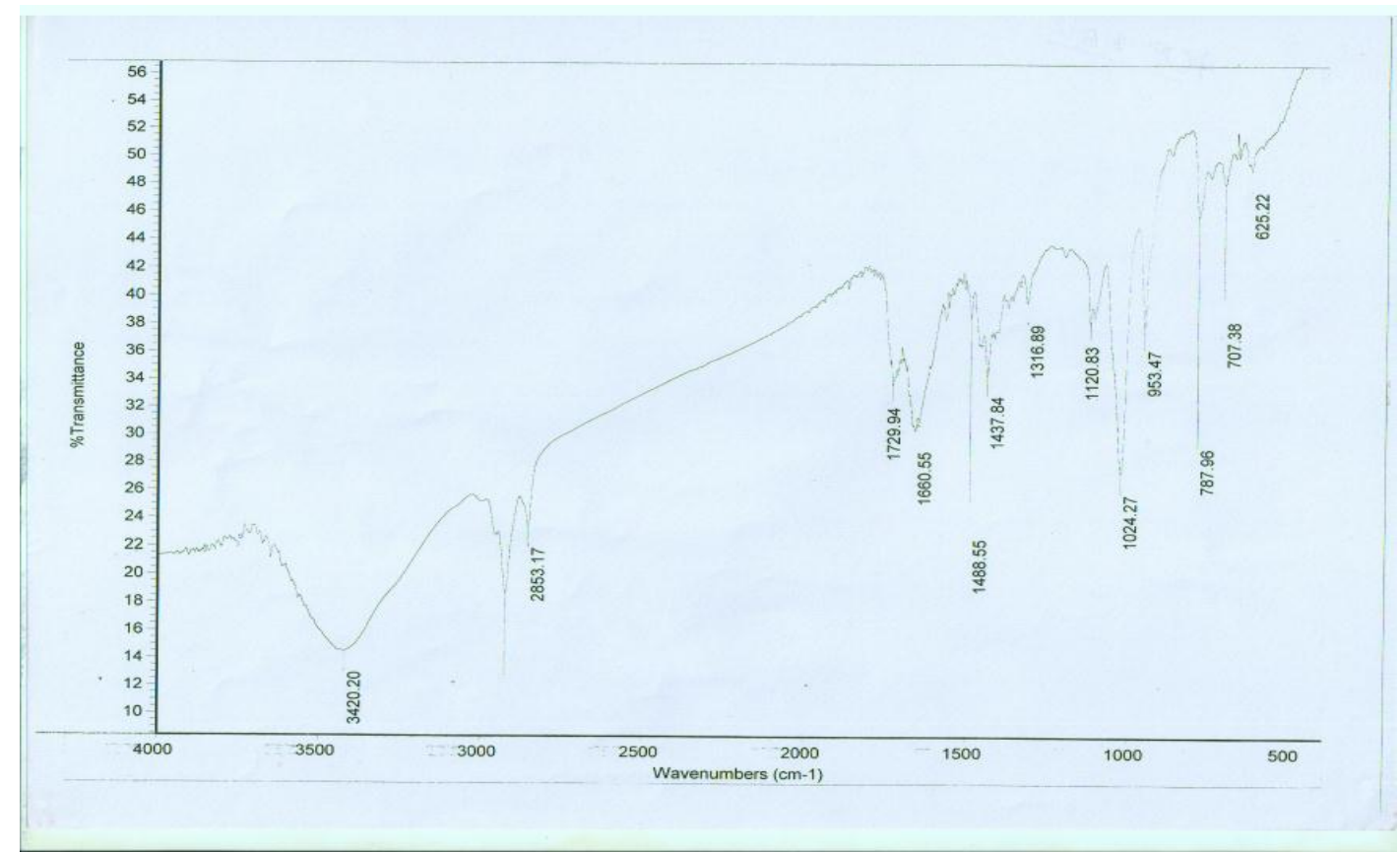

Figure 6:- FTIR spectra of ACE-LEU complex

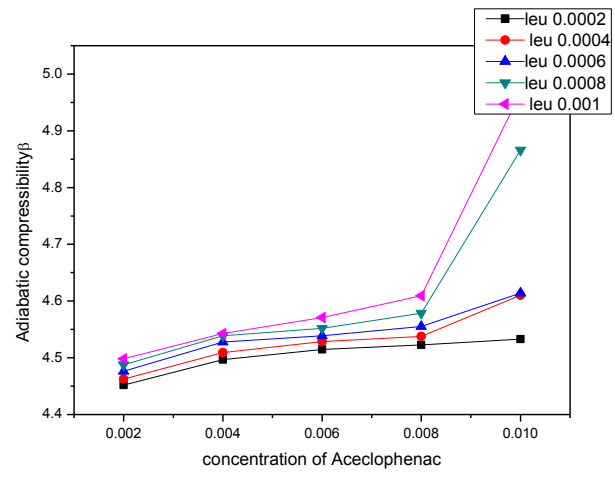

Figure 7:- Variation of adiabatic compressibility with concentration of ACE with varying concentration of LEU

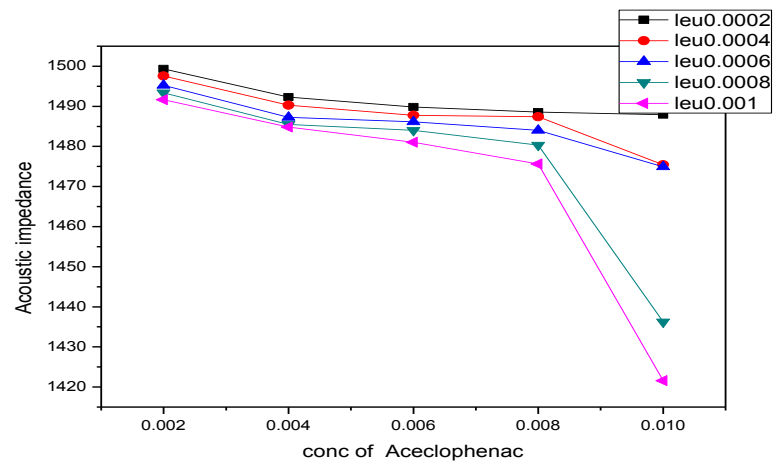

Figure 8:- Variation of acoustic impedance with concentration of ACE with varying concentration of LEU. 


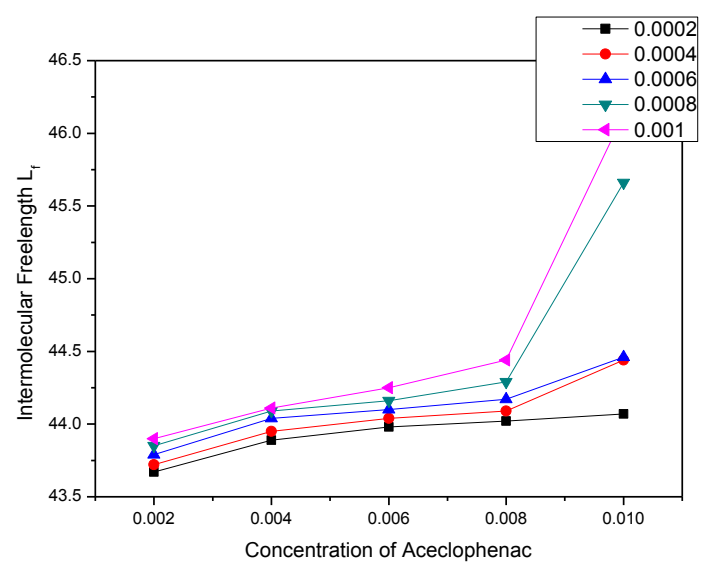

Figure 9:- Variation of Intermolecular free length with concentration of ACE with varying concentration of LEU.

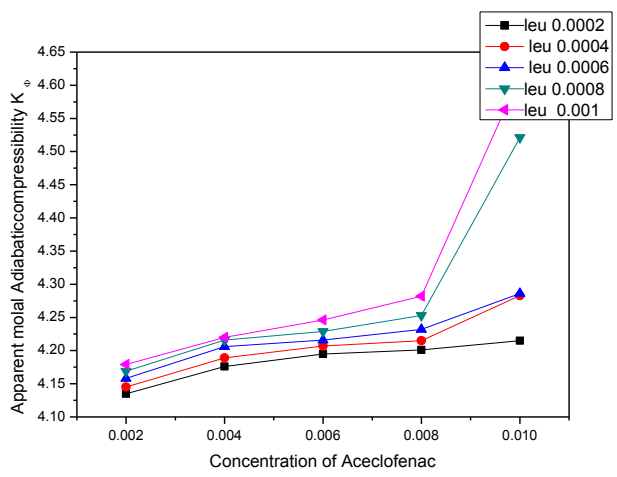

Figure 10:- Variation of apparent molar compressibility with concentration of ACE with varying concentration of LEU

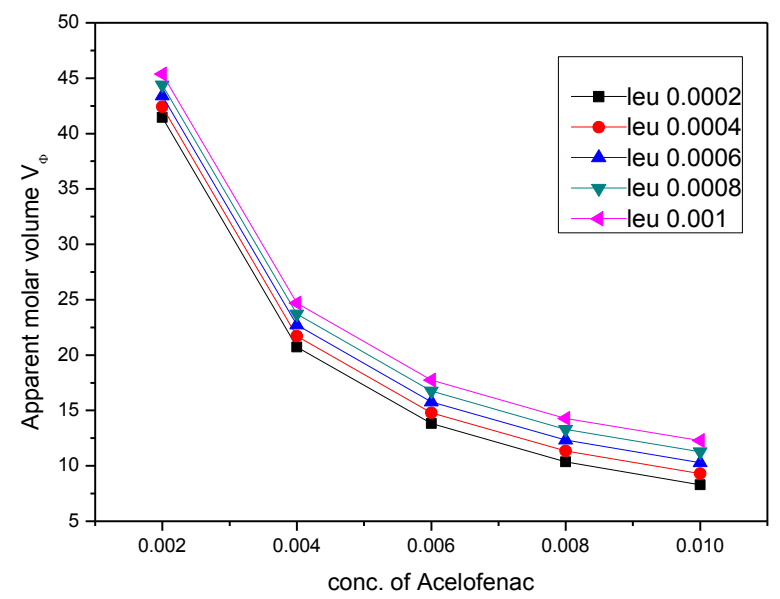

Figure 11:- Variation of apparent molar volume with concentration of ACE with varying concentration of LEU.

\section{Results and Discussion:-}

\section{Spectroscopic study:-}

The molecular interaction between Aceclophenac and Leucine can be interpreted from UV-VIS and FTIR study. The UV-VIS spectra of Aceclophenac,Leucine,(ACE-LEU complex) 
The spectra of Aceclophenac solution was taken in Carry $100 \mathrm{UV}-\mathrm{VIS}$ spectrophotometer. The $\lambda_{\max }$ of Leucine is found at $360 \mathrm{~nm}$. In case Aceclophenac two peaks were observed at $320 \mathrm{~nm}$ and at $450 \mathrm{~nm}$. After mixing both the Leucine peak and one peak of aceclophenac gets completely vanished and a new peak is created at $345 \mathrm{~nm}$. This indicates a considerable interaction between the drug Aceclophenac and Leucine in DMSO medium creating a new amide linkage at hydrophilic end.

IR spectra of LEU, ACE and ACE-LEU complex are represented by Figure 4, 5 and 6 respectively.

The IR data of compounds are assigned in Table 1.

LEU, ACE and ACE-LEU show different IR bands in different regions.

The characteristic band at $2957 \mathrm{~cm}^{-1}$ corresponds to $v_{\mathrm{NH}_{2}}$ as present in LEU. The stretching vibration at $1406 \mathrm{~cm}^{-1}$ and $1581 \mathrm{~cm}^{-1}$ correspond to asymmetric $\left(\boldsymbol{v}_{a s y(c-O-C)}\right)$ and symmetric $\left(v_{\text {sym }(c-O-C)}\right)$. Similarly the band at 3331 $\mathrm{cm}^{-1}$ is an indicative of $v_{O H}$ for ACE. It shows two characteristic bands at $1178-1280 \mathrm{~cm}^{-1}$ and $1050-1178 \mathrm{~cm}^{-1}$ attributed to $v_{s y m(c-O-C)}$ and $v_{a s y(c-O-C)}$ respectively. The absorption band at $1771 \mathrm{~cm}-1\left(v_{C=O}\right)$ for ACE is shifted to lower wave number $1730 \mathrm{~cm}^{-1}$ which indicates the interaction of ACE and LEU. But no peak is observed for carboxylate ion and $-\mathrm{NH}_{2}$ group for ACE-LEU complex indicating the coordination of $-\mathrm{OH}$ group and $-\mathrm{NH}_{2}$. This interaction is confirmed by the appearance of band at $1435 \mathrm{~cm}^{-1}\left(v_{C-N}\right)$ attributed to the formation of amide linkage. It is a clear indication that $-\mathrm{OH}$ group of carboxylate react with $\mathrm{H}$ of $-\mathrm{NH}_{2}$ losing water molecule and formation of amide group. So in product Drug and Leucine interact to form an amide linkage which is supported by FTIR data.

\section{Ultrasonic study:-}

The basic parameters like Ultrasonic Velocity, density, viscosity at various concentrations and temperature of $303.15 \mathrm{~K}$ and other acoustic parameters are calculated using standard formula. The following parameters are calculated using speed of sound, density and other data. The parameters are Adiabatic Compressibility ( $\beta$ ).Apparent molar volume $\left(\mathrm{V}_{\varphi}\right)$.Acoustic Impedance $(\mathrm{Z})$, apparent molar adiabatic compressibility $\left(K_{\phi}\right)$.Intermolecular free length (Lf),Partial molar volume $V_{\phi} \&$ partial molar compressibility. $\left(\mathrm{K}_{\varphi}{ }^{\circ}\right)$. The density, ultrasonic velocity and viscosity are represented in Table 2.The experimental value of adiabatic compressibility, intermolecular free length, apparent molar volume, apparent molar adiabatic compressibility, and acoustic impedance with increasing mole fraction of ACE at different concentration of Leucine is listed in table 3.

The ultrasonic velocity for Aceclophenac in presence of amino acid Leucine shows a decreasing trend (Table-2) at $303.15 \mathrm{~K}$. The variation of $\mathrm{U}$ depends upon concentration of solute and force of electrostriction and temperature. In the presentstudy the value of $U$ constantly decrease as the concentration of drug increased and also with Leucine concentration which indicate drug amino acid interaction in DMSO.As U decreases this indicates the solute is a structure breaker.

When Leucine is added to Aceclophenac solution in DMSO it do not interact with the solvent but the bulk become more structured having less force of electrostriction. This may be attributed to fact that more volume is available for next incoming molecule, so the adiabatic compressibility increases with increase in concentration of aceclophenac as shown in Figure 7.The increase of adiabatic compressibility show quite interesting trend after Aceclophenac concentration reaching $0.008 \mathrm{~m} / \mathrm{dm}^{3}$. The sudden rise in the adiabatic compressibility of both the solute indicates a strong solute-solute interaction and least electrostriction in the medium.

Due to presence of two methyl group in Leucine the electrostriction decrease as a result of hydrophobic salvation. The Acoustic Impedance is calculated using standard relations and found to decrease (Figure 8) which is in good agreement with $\mathrm{U}$, which indicate significant interaction between Aceclophenac and Leucine in DMSO at a higher concentration of solutes. 
The structural change occurring in ternary mixture with increase in concentration may cause the decrease in ultrasonic velocity indicating more Aceclophenac-Leucine interaction. It is observed that $\mathrm{U}$ and $\mathrm{Z}$ show nonlinear decreasing variation with concentration of aceclophenac justify a complex formation between ACE and LEU. $L_{f}$ depends upon the size of the molecules as well as the distance between the surface of molecules. $L_{f}$ values steadily increase with varying concentration of Leucine at lower concentration but sudden rise at high concentration signifies rearrangement of hydrophilic \& hydrophobic moiety.(Figure 9).

The result of the present experiment is in good agreement with Eyring Kincaid model of sound propagation [22]. The theory predicts that ultrasonic velocity decreases with increasing of intermolecular free length in a liquid mixture.

The apparent molal compressibility increases which justify the decrease in ultrasonic velocity as the concentration of ACE increases shown in Figure 10.

The increase in $K_{\phi}$ indicates enhanced molecular association between the Aceclophenac and Leucine with increase of drug as well as Leucine concentration. $K_{\phi}$ and $V_{\phi}$ vary linearly with concentration of ACE (Figure 10 and 11) and it indicates that

$K_{\phi}$ and $V_{\phi}$ values are positive at all concentration of Aceclophenac

The increase in $K_{\phi}$ suggests the increase in ACE and LEU interaction which decrease in caging effect. The decrease in $V_{\phi}$ indicates release of $\mathrm{H}_{2} \mathrm{O}$ molecule through amide linkage formed between $-\mathrm{COOH}$ of $\mathrm{ACE}$ and $-\mathrm{NH}_{2}$ of LEU. (Scheme-I)

At low concentration of both the solutes $V_{\phi}$ values are positive and comparatively higher which means presence of interaction of DMSO with solutes but such interaction get diminished with increasing concentration of Leucine and Aceclophenac indicating decrease in stacking interaction between Aceclophenac and DMSO. The decrease in $V_{\phi}$ indicates release of $\mathrm{H}_{2} \mathrm{O}$ molecule through amide linkage formed between Leucine and aceclophenac and strong ionion attraction such that they occupy less space.

Falkenhagen[23-27] coefficient, $A$ is due to the contributions from inter-ionic forces. The $B$-coefficient of JoneDole equation indicates the ion-solvent interaction in solutions and provides useful primary data about the solvation of ions and their effect on the structure of solvent surrounding the solute molecules and has been interpreted as a measure either of structure forming or structure breaking capacity of a solute in solution depending on its magnitude. These coefficients are calculated by least square method using Jone-Dole coefficient.[28] The values $A$ and $B$ are listed in Table 4.In the presence of Leucine increase in positive values of $A$ indicate solute-solute interaction. It means aceclophenac favourably interacts with Leucine and at its higher concentration interaction becomes more significant by formation of amide linkage and removal of water molecule. The $B$-coefficient values are negative but it decreases with substrate concentration which means Aceclophenac-DMSO interaction get weakened in the comparison of aceclophenac- Leucine interaction.[29] So the interaction of drug- amino acid is much dominating than drug-solvent interaction. The solute-co-solute interaction may occur due to the formation of amide bonding between Zwitter ion of Leucine and hydroxyl (hydrophilic) group of aceclophenac (Scheme-II).

\section{Conclusion:-}

The current experiment can be summarized by the fact that interaction of Aceclophenac with Leucine in DMSO medium can provide various information regarding interaction sites and transformations. The UV-VIS and FTIR spectra conclusively prove interaction of Aceclophenac with Leucine. At low concentration of Aceclophenac and Leucine some association is observed between them but with increase in concentration of drug and amino acid the interaction between them becomes significant and finally association can be interpreted as formation of a new compound which is illustrated in drastic variation of all the parameters at high concentrations. The assumption is again established by the increase in positive values of Falkenhagen coefficient ' $A$ ' with substrate concentration indicating strong solute-solute interaction. The study of kinetics \& pharmaceutical properties of the synthesized product may explore a new horizon in the medicinal chemistry. 


\section{Interaction of Leucine and Aceclophenac:-}
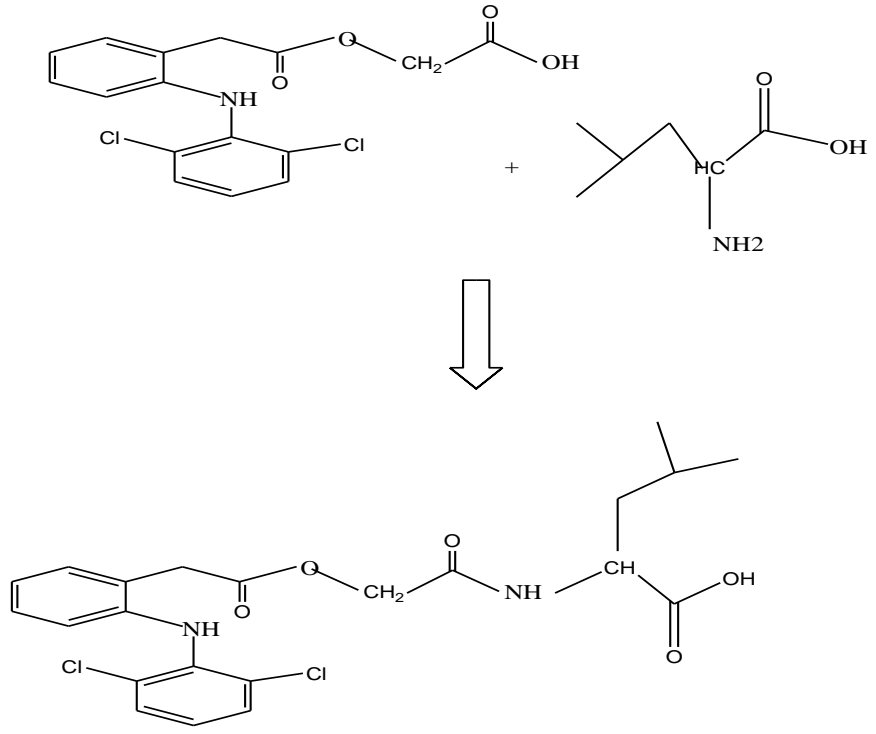

Scheme-I:- Reaction of Aceclophenac with Leucine in DMSO medium.

\section{Hydrophobic, Zwitter ion \& hydrophilic moiety of Leucine and Aceclophenac}

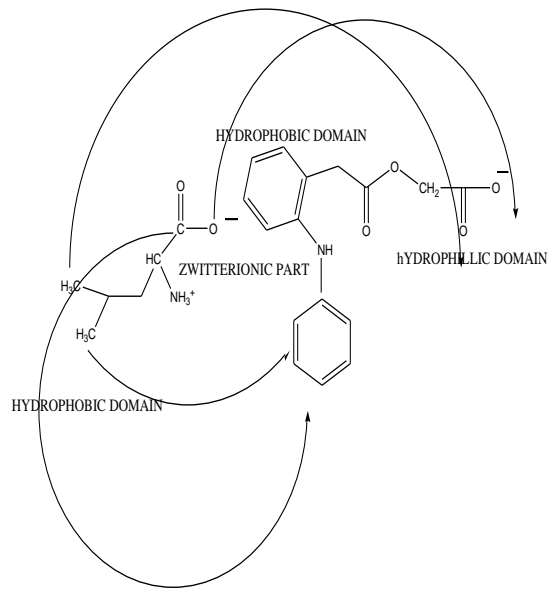

Scheme- II:- Bonding between Zwitter ion of Leucine and hydroxyl (hydrophilic) group of Aceclophenac

\section{Acknowledgment:-}

One of the author Mr S K Pradhan like to acknowledge the UGC for sanctioning the Teacher fellowship to him and Ravenshaw University for providing experimental facilities to undertake the present work.

\section{References:-}

1. Attwood, D., Blundell, R., Mosquera, V., Garcia, M. and Rodriguez, J., (1994): Apparent molar volume and adiabatic compressibility's of aqueous solution of amphiphilic drugs. Colloid and Polymer science, 272, 108104.

2. Kumar, B., Jain, S,K., Prajapati, S, K.(2011): Effect of penetration enhancer DMSO invitro skin permeation of Acyclovir transdermal microemulsion formulation, International journal of drug dlivery,3, 83-94.

3. Bedale, A. R., Shah, R., Narkhede, S. B., Jadav, A. G., Vidyasagar, G.( 2011): Devlopment of characterization of novel aminoacid conjugate of Aceclophenac .International journal of Pharma Tech Research,3(2),841-851.

4. Brad, H. X., Zeiger, W., Suslick, K. S.(2013): Sonochemical synthesis of nano materials .Chem soc Rev,42,2555. 
5. Kharkale, S. S., Bhaskar, C., Agrarwal, P. S., Paliwal, L. M., Nimdeokar, P. G., Effect of solvents on acoustic properties of dihydroformazan and 1,2,4,5 tetrazines at $100 \mathrm{C}$,International journal of EmergingTechnologies in computational and applied Science, ISSN 2279-0047

6. Layman, D. K., Walker, D. K.,Potencial Importance of Leucine in treatmen of Obesity and metabolic syndrome Department of Food Science and human nutrition, Division of Nutrional Sciences, University of illionis, Urbana IL16801

7. 7.Karande, P.,Mitragotri, S.(2009): Enhancement of Transdermal Drug Delivery via Synergistic Action of Chemicals,Biochimica et Biophysica Acta,1788,2362-2373.

8. Thirumaran, S., George, G., Nageswari, G., Satish, K.(2013): 7. Structure making and breaking behaviour of some base aminoacids in aqueous Dimethyl sulphoxides(dmso) medium at Varying temperatures, International journal Research in pure and applied Physics,3(2),10-15.

9. Koly, S. F.,Kundu, S. P.,Kabir, S.,Amran, M. S.,Sultan, M. Z.(2015): Analysis of aceclofenac and bovine serum albumin interaction using fluorescence quenching method for predictive,preventive, and personalized medicine,PMA Journal,6,24.

10. Sathish, R., Ambu, J., Anjana, A., Ahamed, K. F. H. N.,Rao, G. S.(2012): Pharmacokinetic And Pharmacodynamic Interaction Between Aceclofenac And Rosiglitazone In Rats,International Journal of Pharma and Bioscience,3(2),385-394.

11. Wagenmakers, A.J.M.,(1998): Muscle amino acid metabolism at rest and during exercise: role in human physiology and metabolism, Exerc,Sport Sci Rev,26,287-314.

12. Rudreman, N. B.(1975): Muscle Amino Acid Metabolism and Gluconeogenesis,Annual Rev Med,245-258.

13. Sing, V., Chotray, P. K., Gardas, R.(2014): Solvation behaviour and partial molar properties of monosaccharides in aqueous protic ionic liquid solutions, J .Chem thermodynamics, 71,37-49.

14. Pal, A., Chauhan, N.(2012): Interactions of amino acids and peptides with the drug pentoxifyllinein aqueous solution at various temperatures: A volumetric approach, J. Chem. Thermodynamics, 54, 288-292

15. Dhondge, S., Zodape, S., Parwate, D.(2012): Volumetric and viscometric studies of some drugs in aqueous solutions at different temperatures,J Chem Thermodynamics,48,207-212.

16. Galvao, J., Davis, B., Tilley, M., Normando, E., Duchen,M.R., Cordiero, M. F.(2013): Unexpected low dose toxicity of universal solvent DMSO,The FASEB journal,Research communication,28(3),1-14.

17. , V. K., Chauhan, S., Gautam, R.(1998): Ultrasonic velocity measurements of carbohydrates in binary mixtures of DMSO + $\mathrm{H} 2 \mathrm{O}$ at $25^{\circ} \mathrm{C}$,Ultrasonics, 36,619-623.

18. Ibsen, S., Benchimol, M., Esener, S.(2013): Fluorescent microscope system to monitor real-time interactions between focused ultrasound, echogenic drug delivery vehicles, and live cell membranes,Ultrasonics,53,178-184.

19. Fang, J. Y., Hung, C. F., Hua, S. C., Hwang, T. L.(2009): Acoustically active perfluorocarbon nanoemulsions as drug delivery carriers for camptothecin: Drug release and cytotoxicity against cancer cells,Ultrasonics,49,39-46.

20. Xiang, X., Tang, Y., Leng, Q., Zhang, L., Qiu, L.(2016): Targeted gene delivery to the synovial pannus in antigen-induced arthritis by ultrasound-targeted micro bubble destruction in vivo, Ultrasonics,65,304-314.

21. Sinisterra, J. V.(1992): Application of ultrasound to biotechnology: an overview, Ultrasonics,30(3),180-185.

22. Erying, H., Kincaid, J. F.(1938): Free Volume and Free angle ratio of molecules in liquids ,J Chem Physics, 6,620-629.

23. Robinson, R.A., Stokes, R.H.(1959): Electrolytic solutions, Butterworth scientific publications, London.

24. Jones, G., Dole, M.(1929): The viscosity of aqueous solutions of strong electrolytes with special reference to barium chloride, J. Am. Chem. Soc.,51, 2950-2964.

25. Falkenhagen, H., Dole, M.(1929): Viscosity of electrolyte solutions and its significance to the Debye theory, Zeitschrift Für Physik, 30, 611- 616.

26. Feakins, D., Freemantle, D. J., Lawrence, K. G.(1974): Transition state treatment of the relative viscosity of electrolytic solutions. Applications to aqueous, non-aqueous and methanol + water systems, J. Chem. Soc., Faraday Trans., 70, 795-806.

27. Iqbal, M. J., Chaudhry, M. A.(2009): Thermodynamic study of three pharmacologically significant drugs: Density, viscosity, and refractive index measurements at different temperatures. J. Chem. Thermodynamic . , 41, 221-226.

28. Das, M.,Das,S.P.,Patnaik, A.K.(2013): Molecular Interionic Interaction Studies of Benzimidazolium Dichromate and 2-Methyl Imidazolium Dichromate in Water and DMSO + Water at Different Temperatures, Journal of Physical Science, 24(1), 37-50.

29. Zhao, H.(2006): Viscosity B-coefficient and standard partial molar volumes of amino acids, and their roles in interpreting the protein (enzyme) stabilization,Biophys. Chem., 122, 157-183. 